\title{
„Ringen um angemessenes Verhalten“ - Herausforderungen im Internationalen Freiwilligendienst
}

\author{
Dr. Katharina Mangold \\ Wissenschaftliche Mitarbeiterin Stiftung Universität Hildesheim \\ Sozial-und Organisationspädagogik | katharina.mangold@uni-hildesheim.de
}

\section{Zusammenfassung}

Der Beitrag beschäftigt sich mit jungen Erwachsenen aus Deutschland, die einen Internationalen Freiwilligendienst in Uganda absolvieren. Anhand der ethnographischen Studie kann herausgearbeitet werden, dass die Freiwilligen insbesondere herausgefordert sind, sich im Bezug zu Statusinkonsistenz zu positionieren, und häufig nicht wissen, wie sie sich ,angemessen“ verhalten sollen. Genau in diesem Nichtwissen liegt jedoch eine Chance zur Reflexion und zu Bildungsprozessen - so die grundlegende These des vorliegenden Beitrags.

Schlagwörter: Ethnographische Studie, Internationaler Freiwilligendienst, Herausforderungen, Statusinkonsistenz, Jugend

\begin{abstract}
This article focuses on young adults from Germany doing an International Voluntary Service in Uganda. The ethnographic study shows that volunteers struggle to do the right things and with their own status position. However, this kind of struggling can be seen as an opportunity for reflexion and educational processes - so the thesis of the present article.

Keywords: Ethnographic study, International Voluntary Service, challenges, status inconsistency, youth
\end{abstract}

\section{Problemaufriss}

Immer mehr junge Erwachsene in Deutschland nutzen die Möglichkeit, nach dem Schulabschluss Erfahrungen im Ausland zu sammeln und sich freiwillig in sozialen Projekten und Einrichtungen zu engagieren. Waren allein über 30.000 Freiwillige über das Programm Weltwärts des Bundesministeriums für wirtschaftliche Zusammenarbeit und Entwicklung (BMZ) in den letzten zehn Jahren im Ausland, so kommen über das Freiwillige Soziale Jahr (FSJ) im Ausland, den Internationalen Jugendfreiwilligendienst (IJGD) oder auch das Freiwilligenprogramm des Auswärtigen Amtes kulturweit eine Vielzahl hinzu.

Studien im Themenfeld Internationale Freiwilligendienste (IFD) beschäftigen sich häufig mit den Motiven junger Menschen (Krettenauer/Gudulas 2003), nehmen die Wirkung von Internationalen Freiwilligendiensten in den Blick (bspw. Fischer 2011), beschäftigen sich aus einer postkolonialen Perspektive mit der Thematik (bspw. Kontzi 2015) und stellen - wenn auch nur vereinzelt - die Aufnahmeorganisationen ins Zentrum der Betrachtung (bspw. Schwinge 2011). Die Alltagssituationen der 
Freiwilligen vor Ort und die spezifischen mit ihrer Arbeits- und Lebenssituation verbundenen Herausforderungen gelangen bisher wenig in den Fokus. Genau an diesem Punkt setzte die ethnographische Studie „Inbetweenness. Jugend und transnationale Erfahrungen“ (Mangold 2013) an. Zwischen Oktober 2009 und Januar 2010 wurden junge Menschen aus Deutschland, die ihren Freiwilligendienst in Uganda absolvierten, ethnographisch begleitet und interviewt. Die jungen Freiwilligen waren zum Zeitpunkt der Erhebung zwischen drei und sechs Monaten in Uganda und arbeiteten in sozialen Projekten (z. B. Schulen, Krankenhäuser, Einrichtungen für Menschen mit Behinderung). Ausgehend von der Frage „Wie gestalten junge Erwachsene ihren Alltag im Internationalen Freiwilligendienst und welche Erfahrungen machen sie dort?"wurden Beziehungskonstellationen, Freizeitaktivitäten, Arbeitsbedingungen, Wohnsituationen - kurzum, der Alltag der jungen Menschen ethnographisch analysiert. Durch das Dabeisein am Geschehen kann Ethnographie „unter Reflexion des vorgängigen eigenen alltäglichen Verstehens, natürliche, settings' $[$...] beschreiben, um Alltagserklärungen und Alltags-Handeln verstehen zu können“ (Honer 1994: 87; Hervorhebungen im Original). Im Rahmen der Teilnehmenden Beobachtung (Lüders 2003) stand stets das Handeln der Freiwilligen im Beobachtungsfokus, welches detailliert von der Beobachterin in über 200 Seiten verschriftlicht wurde. Diese sogenannten Beobachtungsprotokolle wurden im Anschluss über das kodierende Verfahren der Grounded Theory (Strauss/Corbin 1996) analysiert.

In diesem Artikel wird auf die Herausforderungen im Alltag der jungen Erwachsenen fokussiert und die zentrale Herausforderung als „Ringen um angemessenes Verhalten" rekonstruiert. So soll zunächst aufgezeigt werden, inwiefern sich diese Herausforderung zeigt (Punkt 2) und welche Strategien die jungen Menschen entwickeln bzw. besitzen, damit umzugehen (Punkt 3). Abschließend soll die Ambivalenzsituation „Inbetween“ als Möglichkeitsraum diskutiert werden (Punkt 4).

\section{Zentrale Herausforderung im Internationalen Freiwilligendienst}

Beschäftigt man sich mit Herausforderungen im Internationalen Freiwilligendienst, so werden zunächst Herausforderungen wie der Umgang mit der neuen Sprache, das Zurechtfinden in der „Fremde“ oder der Abnabelungsprozess von den Eltern thematisiert (Mangold 2013).

In den folgenden Ausführungen soll auf eine der meines Erachtens zentralen Herausforderungen im Internationalen Freiwilligendienst eingegangen werden: nämlich das „Ringen um angemessenes Verhalten“. Das erste Beispiel macht dieses Ringen in Alltagssituationen und dem Umgang mit arm und reich, mit Zugehörigkeit und Nichtzugehörigkeit deutlich (2.1). Im zweiten Beispiel wird auf die Statusambivalenz der jungen Menschen eingegangen, die sich sowohl aus dem neu zugeschriebenen Status durch den Ortswechsel bedingt, als auch im alltäglichen 
Handeln reproduziert und hergestellt wird (2.2). In beiden Beispielen wird das „Ringen“ der jungen Menschen deutlich und so kann als zentrale Herausforderung der Umgang mit Ambivalenzsituationen benannt werden.

\subsection{Ringen um angemessenes Verhalten I: „Jedes Handeln ist schwierig“"}

Wie im folgenden Beispiel ausgeführt wird, ist es Jakob - ein 19-jähriger Freiwilliger - nur möglich zu artikulieren, welches Verhalten er für „falsch“ hält und sich davon abzugrenzen. Eine Lösung kann er nicht anbieten, weil er selbst nicht weiß, wie man sich „richtig“ verhalten könnte. Damit drückt er aus, dass jedes Handeln „schwierig“ ist.

Jakob berichtet über eine Europäerin, die sein möchte wie die Menschen in Uganda - „wahrscheinlich würde sie sich auch noch die schwarze Hautfarbe wünschen, wenn das ginge“. Dieses Verhalten definiert Jakob als „going native“ und grenzt sich davon ab:

Klar lerne er auch ein bisschen Luganda, klar passe er sich auch mal an, aber man kann hier ein Jahr sein oder 20 Jahre, man wird nicht so sein wie die, man wird nicht ganz dazugehören. Und dieses ,going native“ wäre auch für die Einheimischen nicht gut. Er erzählt von einer Finnin, die ein Dorf weiter lebt. Es gab die Situation, dass Jakob von einem Mann angebettelt wurde und er zunächst Geld und dann ein Chapati [ugandisches Fladenbrot] von ihm wollte. Jakob wollte ihm das aber nicht geben. Dann kam die Finnin und wies den Mann auf Luganda zurück. Die Finnin hätte auch immer afrikanische Kleider an. Jedenfalls wies sie den Mann zurück, der sie darauf hin nur sehr sehr abfällig nachgeäfft hat. Man könne also mit dem Soseinwollen wie die Einheimischen auch nichts erreichen, das sei eher noch schwieriger.

Jakob grenzt sich nicht komplett ab, sondern macht Zugeständnisse, indem er betont, dass auch er „ein bisschen Luganda“ lerne, dass auch er sich mal anpasse. Es ginge nicht (immer) darum, das „krasse Gegenteil“vom „going native“ zu sein. Jakob argumentiert nicht nur für sich oder für andere Europäer_innen, sondern bezieht hier die ugandische Bevölkerung in seine Überlegungen mit ein und unterstreicht damit, dass man sich von „going native“ abgrenzen müsse, weil es niemandem nützlich sei.

In der Praxis des Bettelns wird eine Differenz hergestellt, nämlich die des Besitzenden und die des Besitzlosen. Zu dieser Differenz muss sich Jakob verhalten. Wir erfahren nicht, wie Jakob sich verhält, ob er zögert, überlegt oder sofort agiert, wir erfahren lediglich, dass Jakob „ihm das aber nicht geben“ will. Sein Verhalten wird von ihm als angemessen ausgewiesen, was ein Gegensatz zu der Darstellung des Verhaltens der Finnin bedeutet. Sie nämlich verhält sich „falsch“. Sie „wies den Mann auf Luganda zurück“. Durch die Zurückweisung auf Luganda wird der 
Mann, der zunächst der Bettelnde und somit auch Bedürftige ist, zum Richter der Szene. Er äfft die „Finnin“ nach und zwar „sehr sehr abfällig“. Er stellt sich damit auf die Seite von Jakob und positioniert sich ebenfalls gegen das „going native“.

Jakob lässt keinen Zweifel, so wie die „Finnin“ sich verhält, ist es falsch, aber wie es richtig wäre sich zu verhalten, darauf scheint er selbst keine Antwort zu haben. Dieses Dilemma drückt sich vor allem darin aus, dass er zwar deutlich betont, dass man „mit dem Soseinwollen wie die Einheimischen auch nichts erreichen“ könne und dass dies „eher noch schwieriger“ sei. Das „noch“ lässt darauf schließen, dass auch das andere Verhalten und somit sein Verhalten „schwierig“ ist, dass es also gar kein nichtschwieriges Verhalten gibt. Er kennt es zumindest nicht und kann nicht sagen, wie es richtig ist, er kann sich nur von dem abgrenzen, was er als „noch schwieriger" definiert. Dahinter steckt, dass man es nicht richtig machen kann, egal was man tut, es ist schwierig. In diesem Dilemma steckt Jakob mit seinem Verhalten und Handeln in Uganda und sieht keinen Ausweg.

\subsection{Ringen um das angemessene Verhalten II: Statusambivalenzen}

Die jungen Menschen positionieren sich nicht nur durch ihr Handeln und ihre Äußerungen selbst, sie werden auch im Handeln von anderen positioniert. Es handelt sich also um einen interaktiven Herstellungsprozess. Dies konnte am Beispiel von Jakob aufgezeigt werden. Der Freiwilligendienst an sich ist jedoch nochmals gerahmt von einer strukturellen Positionierung des oder der Freiwilligen: Die jungen Menschen gehen mit deutschem Pass (oder zumindest vergleichbarem Aufenthaltsstatus in Deutschland) nach Uganda, zudem handeln sie als „Weiße“, sie haben alle einen Schulabschluss (meist Abitur) in Deutschland gemacht, kommen meist aus Mittelschichtfamilien und möchten nach ihrem Auslandsaufenthalt häufig ein Studium beginnen. Sie handeln also als Privilegierte, die sich einen Freiwilligendienst „leisten“ können und denen ein Freiwilligendienst „ermöglicht“ wird. In dieser Position beginnen sie ihren Freiwilligendienst, woraus sich Herausforderungen ergeben, die sie in ihrem bisherigen Leben wenig erlebt haben.

Anhand der Wohnsituation von Christina und Michael soll dieser Frage nach dem „zugewiesenen“ Status und den damit verbundenen Positionen und (Selbst-)Positionierungen nachgegangen werden. Auch dabei lassen sich (Status-)Ambivalenzen erkennen, die ebenfalls mit dem Muster „Ringen um angemessenes Verhalten“ überschrieben werden können.

Michael und Christina - beide nach Ende ihres Bachelorstudiums - erleben eine Art „Statusaufstieg“, als sie nach dem Wohnen in einer gemeinsamen Studenten-WG in Deutschland plötzlich in einem eigenen Haus in Uganda leben - „Es sei schon krass: ,Wir sind grad mal 24, haben grad fertigstudiert und jetzt wohnen wir in einem Haus in Uganda،“" Der Ortswechsel von Deutschland nach Uganda 
verändert ihre finanzielle Lebenssituation. Nicht weil sie plötzlich reich seien, sondern weil in Uganda mit weniger Geld mehr Wohlstand möglich sei. Es kommt somit auf den jeweiligen Kontext an, ob die Freiwilligen als eher wohlhabend oder eher bedürftig definiert werden und sich dann weiter auch in dieser Position selbst verorten. Michael definiert diese Situation selbst als „krass“ und drückt damit möglicherweise einerseits Begeisterung aus, andererseits wird darin deutlich, dass er dazu ein ambivalente Haltung hat. Er fühlt sich nicht ganz wohl dabei und ist um ein angemessenes Verhalten bemüht. Er fühlt sich „komisch“, dass er einen Gärtner hat, sieht jedoch auch keinen anderen Weg:

Leute, die seinen Garten richten, das sei schon auch komisch, so sei er ja eigentlich gar nicht, aber hier kann man es sich halt leisten und wäre alleine auch über zwei Tage beschäftigt den eigenen Rasen zu mähen.

Michael fühlt sich nicht wohl in der Situation und er artikuliert „es sei schon auch komisch“. Gleichzeitig distanziert er sich von seinem Handeln bzw. distanziert sein aktuelles Handeln von seinen Charakterzügen, weil „so sei er ja eigentlich gar nicht“. Er verhält sich also in Uganda aktuell so, wie er ,eigentlich“ gar nicht ist. Er nimmt Bezug auf die Rahmenbedingungen, indem er einerseits argumentiert, dass er alleine zwei Tage dafür benötigen würde, um den Rasen selbst zu mähen, und andererseits, dass die Arbeitskräfte in Uganda so günstig seien, dass „man“- bzw. im konkreten Fall eben er - sich das leisten könne. Er differenziert zwischen den Bedingungen in Deutschland. Dies wird zwar nicht explizit angesprochen, doch in dem „hier“ wird gleichzeitig auch ein „dort“ hergestellt. Dieses „dort“ muss Deutschland sein. Da er in Uganda andere Rahmenbedingungen vorfindet, handelt er auch anders. Dabei fühlt er sich „schon auch komisch“.

Zusammenfassend kann festgehalten werden, dass sich die Freiwilligen aufgrund des Ortswechsels in neuen Lebenssituationen befinden, in denen ihnen einerseits ein neuer bzw. anderer Status als bisher in Deutschland zugeschrieben wird und sie sich selbst auch in dieser neuen Statusposition anders bewegen (müssen). Die Inkonsistenz der eigenen Statusposition ergibt sich aber nicht nur aufgrund der unterschiedlichen Statuspositionen in Uganda und Deutschland, sondern auch in Situationen des Hin-und-Hergerissen-Seins in Handlungssituationen in Uganda.

\subsection{Bündelung: Herausfordernde Ambivalenz „Inbetween“?}

Diese Herausforderungen (nicht zu wissen, wie man sich verhalten soll und der Umgang mit der Statusinkonsistenz) „dramatisieren“ sich in der Situation der Transnationalität und im Kontext des jungen Erwachsenenalters, weil hier Verlässlichkeiten qua Lebensphase (Jugend) oder Lebenssituation (im Transnationalen) wenig gegeben sind (Mangold 2013). So müssen die Freiwilligen nicht nur mit dem Übergang von der Wohnsituation bei ihren Eltern in eine eigene Wohnung 
zurechtkommen, sondern sie leben außerdem in einem „eigenen Haus“ und haben „Hausangestellte“, was sie (zunächst) nicht mit ihrem „Jugendstatus“ in Einklang kriegen. Jakob beispielsweise agiert im Spannungsfeld arm-reich, wenn er davon berichtet, wie er als junger europäischer Mann von einem ugandischen Mann angebettelt wird. So wird er vom Abiturienten zum Wohlhabenden gemacht. Die neue Situation im Ausland fordert die Freiwilligen kontinuierlich auf, sich in ihrem Handeln und Reden zu sich selbst und zu den sozialen Situationen zu positionieren. Dabei „ringen“ sie immer wieder um das „angemessene“ Verhalten. Dieses Ringen kann als Ausdruck einer Ambivalenzsituation interpretiert werden. In diesem Zusammenhang wird deutlich, dass es sich um eine „unauflöslich[e] und unlösbar[e]“ (Lüscher 2000: 98) Situation handelt, welche mit polarisierenden Handlungs- und Denkmustern verknüpft ist und eben gerade nicht einseitig aufgelöst werden kann. Diese Ambivalenz drückt sich im „Ringen um angemessenes Verhalten“ aus und stellt die jungen Erwachsenen im Internationalen Freiwilligendienst vor eine prägende Herausforderung. Wie aber gehen die Freiwilligen mit dieser Herausforderung um? Mit dieser Frage beschäftigt sich der folgende Abschnitt.

\section{Umgangsformen mit den herausfordernden Situationen}

Betrachtet man das Datenmaterial, so lassen sich zwei Formen des Umgangs mit herausfordernden Situationen des „Ringens um angemessenes Verhalten“ rekonstruieren.

(1) Erstens versuchen die jungen Menschen sich einem „einfachen“ (wenig komplexen) Ordnungsschema zu bedienen. Dieses ist häufig an ihr bisheriges Denken und an ihre bisherigen Bilder anschlussfähig. Allzu lange haben sie ein „Afrikabild“ vermittelt bekommen, das beispielsweise mit Armut verknüpft ist. Das bipolare Denk- und Handlungsschema, auf welches die jungen Menschen zurückgreifen, schafft ihnen dabei Sicherheit und Ordnung. Es erspart ihnen, jedes Mal von neuem entscheiden zu müssen, sondern es gibt einen Erklärungsansatz und eine Handlungsorientierung.

(2) Als zweite Möglichkeit, Sicherheit zu erzeugen, bietet sich der Zusammenschluss in der Freiwilligengruppe an. Hier schaffen sich die Freiwilligen einen Raum, in welchem sie sich nicht erklären müssen, in welchem sie sich mit ihrem Handeln und Denken sicher, vertraut und verstanden fühlen.

Im Folgenden soll nun anhand zwei empirischer Beispiele auf diese beiden Umgangsformen mit der herausfordernden Ambivalenzsituation eingegangen werden.

\subsection{Umgangsform I: Bipolare Differenzkonstruktion}

In der folgenden Szene läuft die Beobachterin mit Jakob und Markus [zwei Freiwillige] zur Schule, in welcher die beiden arbeiten. Die beiden jungen Männer leben gemeinsam mit drei Priestern in einer Kirchengemeinde in einem Dorf. Sie haben 
sich gerade mit einem der Priester unterhalten, der mit einer Autobatterie eine Vorrichtung für eine Glühbirne baut:

Dann laufen wir weiter. Die beiden unterhalten sich darüber, dass in Deutschland kein Pfarrer auf die Idee kommen würde, ein elektrisches Gerät zu bauen, ja dass in Deutschland eh immer jeder einen Handwerker kommen lässt, wenn irgendwas nicht funktioniert. 90\% der Deutschen würden das mit Sicherheit so machen. Aber hier sei das anders, vielleicht auch deshalb, weil wenn man hier ein Problem hat und dann jemand anruft, um zu fragen ob er hilft, dann wartet man erstmal ewig und wenn man keine Lust hat tagelang zu warten, dann muss man es eben selbst machen. Die Jungs lachen. Ja das sei sogar so, wenn man vom parish [der Kirchengemeinde] die Polizei im Dorf rufen würde. Die Fathers haben gesagt, dass wenn hier ein Überfall wäre, dann könnte man nicht auf die Polizei warten, die kommen viel zu spät. Jakob erzählt, dass die Polizei in Dorf nicht mal ein eigenes Auto hat, sie müssten sich also mit dem Taxi hierherfahren lassen. Die beiden lachen und Markus setzt eines drauf, indem er sagt, dass das Taxi aber leider noch nicht voll genug sei und sie noch auf weitere Mitfahrende warten müssen.

In dieser Szene wird ein „hier“ und ein „dort“ hergestellt. So wird „Deutschland“ als Ort markiert, in dem „eh immer jeder einen Handwerker kommen lässt, wenn etwas nicht funktioniert“. „Die Deutschen“ kümmern sich demnach nicht selbst darum, sondern fragen eine Dienstleistung ab. Diese Verallgemeinerung wird im Folgenden nochmal etwas relativiert, indem zumindest zehn Prozent der Deutschen ausgenommen werden. Die zwei jungen Männer reden so über „die Deutschen“, als ob sie selbst nicht dazugehören würden und sich davon distanzieren, vielleicht möchten sie sich von den 90 Prozent abgrenzen, die es „mit Sicherheit so machen“. Sie distanzieren sich von der Masse und ermöglichen sich selbst, etwas Besonderes zu sein. In dem „einen“ Bild nämlich alle Deutschen holen sich Hilfe - finden sie sich selbst nicht wieder und müssen darum eine Möglichkeit für mehr Vielfalt finden, damit sie sich selbst weiterhin als „Deutsche“ verorten und sich zugleich von der Masse abgrenzen können. Sie schaffen somit ein Zugehörigkeitskriterium zu einer Nationalgruppe und gleichzeitig eine Differenzierungslinie zu dieser und können sich darum als etwas Besonderes generieren. Hierdurch konstruieren sie Vielfältigkeit zu einer zunächst homogenisierende Herstellung von „den“ Deutschen bzw. der Praxis aller Deutschen. Nachdem sie nun das Verhalten „der Deutschen“ reflektiert haben, wechseln sie die Perspektive und können - indem sie nun auch das Leben in Uganda kennen - sagen, wie sich eine Situation in Uganda abspielt, wenn etwas kaputt geht. Zunächst betonen sie deutlich die Differenz: „Hier sei das anders“. Sie beziehen also „hier“ (Uganda) und „dort“ (Deutschland) aufeinander und stellen einen Vergleich her. Dieser Vergleich führt zur Differenzierung. Nun versuchen sie, Gründe zu finden, warum es „hier“ anders ist als „dort“, und begründen dies mit der gemachten Beobachtung in Uganda, dass man hier „ewig“ warten muss, bis jemand kommt, um etwas zu reparieren und dass man es letztlich 
selbst machen muss, wenn man nicht tagelang warten will. Es ist also ein Handeln aus der Not heraus. Das Verhalten „der Ugander*innen“ wird jedoch nicht binnendifferenziert, sondern die Konstruktion von Uganda bzw. dem Verhalten „der Ugander*innen“ erfolgt als homogenes Bild. Mit dem weiteren Beispiel des Polizeieinsatzes untermalen sie ihre Beobachtung und ihre Zuschreibung von „den Ugander*innen“.

Anhand dieser Szene lassen sich meines Erachtens zwei Aspekte festhalten: (1) Durch das Inbezugsetzen von Erfahrungen aus ihrem Heimatland mit denen ihres Aufenthaltes in Uganda wird durch Rückgriff auf die Kategorie von Nationalstaaten ein „hier“ und ein „dort“ konstruiert. Dieses „hier“ und „dort“ wird dann mit spezifischen Zuschreibungen verknüpft, welche zu einem Bild führen. (2) Das eine Bild, welches entworfen wird, reicht ihnen aber in den Zuschreibungen zu „den Deutschen“ nicht aus, weil sie sich selbst darin nicht wiederfinden und abgrenzen wollen, aber dennoch „Deutsche“ sind/sein wollen. Die bipolaren Zuschreibungen erzeugen also (scheinbare) Klarheit und Sicherheit, sind aber - so das Beispiel - zu sehr komplexitätsreduzierend.

\subsection{Umgangsform II: Gesellung unter "Gleichen“}

Als zweite Handlungsstrategie im Umgang mit herausfordernden Ambivalenzsituationen soll der Blick nun auf die Gesellungsformen der jungen Menschen im Internationalen Freiwilligendienst gerichtet werden. Mit wem verbringen die Freiwilligen also ihre Zeit? Wo und mit wem fühlen sie sich wohl und tauschen sich aus?

Dabei kann zunächst festgehalten werden, dass die Freiwilligencommunity selbst einen wichtigen Ort des Austausches und der Freizeitgestaltung darstellt. Hier werden Sorgen geteilt, gemeinsame Zeit verbracht und vieles mehr. Ein weiterer Blick in die Daten zeigt jedoch, dass die Freiwilligencommunity, die sich zunächst über die Zugehörigkeitskriterien Alter, Freiwilligenstatus, Bildungsstand und Hautfarbe konstruiert, Erweiterung findet. Dies kann das folgende Beispiel deutlich machen, in welchem es um Michael (Freiwilliger) geht, der krank zu Hause ist und von Paul, einem deutschen Entwicklungshelfer, besucht wird. Als Paul seinen Besuch beendet, bleiben Michael und die Beobachterin im Esszimmer sitzen und unterhalten sich:

Michael erzählt von seinem Bild der Ugander und sagt, dass die halt meistens „über Arbeit, über Geld und wie schwer das Leben einfach ist - das geht mir halt einfach manchmal auf den Sack“. Darum hat er sich auch total geärgert, dass er gestern noch so unfit war, weil sie bei den weißen Brüdern eingeladen gewesen sind. Das sind deutsche Priester, die schon lange hier leben und mit denen man halt mal richtig reden kann. Einer ist ehemaliger Philosophieprof, mit dem könnte man halt auch mal über Gott und die Welt philosophieren. Er kenne die nur über Paul und es sei nicht so, dass man da einfach vorbeigehen kann, aber er hätte wirklich mal Lust sich mit denen zu unterhalten (BP 36, Zeile 8-26). 
Michael thematisiert seine Beziehung zu „den Ugandern“ und seine Beziehung zu „den deutschen Priestern“. Dabei kreiert er ein Bild von „weißen Brüdern“, mit denen wirkliche Verständigung stattfindet und von „Ugandern“, mit denen es oftmals schwierig zu reden ist. Michael relativiert seine Aussage über „die Ugander“ zwar mit den Begriffen „meistens“ und „manchmal“, aber er ist dennoch sehr klar und vehement in seinem Urteil. Er fügt dieses Bild über die „Ugander“ ein, um den Kontrast zu verdeutlichen, wie es mit „Deutschen“ ist oder wäre, „mit denen man halt mal richtig reden kann“. Die „Ugander“ dienen somit als Kontrastfolie für die „deutschen“ Priester. Unter „richtig reden“ versteht er „,̈̈ber Gott und die Welt philosophieren“, dahinter steckt ein Verlangen nach tiefer Verständigung und Austausch. Auch verbirgt sich darin die implizite Vermutung, dass Deutsche, die in Uganda leben, ähnliche Erlebnisse und Erfahrungen machen wie er selbst. Das Verb „philosophieren“ deutet auf etwas Exklusives hin: Man nimmt sich Zeit zum Philosophieren und diskutiert „über Gott und die Welt“. Hier werden nicht Alltagsprobleme verhandelt, sondern hier lässt man seinen Gedanken freien Lauf. Michael findet es schwierig, dass es bei „den Ugandern“ immer nur um Armut und Krankheit geht, er wünscht sich etwas anderes, es geht ihm „auf den Sack“. Er empfindet diese Situation als Belastung und nimmt an, dass er mit den „weißen Priestern“ andere Themen diskutieren kann. Er freut sich auf nahezu Unbekannte, aber sie sind ihm doch vertrauter als „die Ugander“. Der Kontakt zu den „weißen Priestern" ist ein Wunsch von Michael, der noch nicht verwirklicht wurde. Daher ärgert er sich, diese Möglichkeit aufgrund seiner Krankheit zu verpassen.

Anhand der Szene wird deutlich, dass Michael sich von „vermeintlich Gleichen“ angezogen fühlt, da er hier echtes Verständnis vermutet. Die Gesellungsform erweitert sich hier über den Status Freiwillige hinaus, ebenso wie über verschiedene Generationen hinweg. Die angenommene ähnliche Lebenssituation - nämlich als Deutscher in Uganda zu leben - wird als verbindend gewertet. Die Zuschreibung zu „den Ugandern“ fällt hingegen äußerst negativ aus. Dennoch lassen sich an anderen Textstellen im Datenmaterial Freundschaftsbeziehungen mit Ugander*innen rekonstruieren. Die genauere Analyse macht deutlich, dass hier die Konstruktion „beide Welten kennen“ und „ähnlicher Bildungsstatus“ die jeweiligen Zugehörigkeitsmerkmale herstellen, da es sich hier häufig um Ugander*innen handelt, die bereits Zeit in Europa verbracht haben und einen ähnlichen Bildungsabschluss wie die Freiwilligen selbst vorweisen können.

\subsection{Bündelung: Erfolgreiche Strategien des Umgangs mit herausfordernden Ambivalenzsituationen}

Die beiden Umgangsformen „bipolare Differenzkonstruktionen“ und „Gesellungsformen unter Gleichen“ entlasten die jungen Freiwilligen und stellen daher eine erfolgreiche Strategie im Umgang mit herausfordernden Ambivalenzsituationen 
dar. Durch klare bipolare Deutungs- und Erklärungsmuster müssen sie nicht ständig ihr Handeln infrage stellen und neu entscheiden. In der Community unter Gleichen fühlen sie sich verstanden und gelangen weniger in Situationen, in denen sie nicht wissen, wie sie agieren sollen.

\section{Fazit: „Inbetween“ als Chance?}

Die aufgezeigten Umgangsweisen mit herausfordernden Ambivalenzsituationen machen zunächst deutlich, dass es den jungen Erwachsenen im Internationalen Freiwilligendienst gelingt, Möglichkeiten zu finden, die sie in dieser herausfordernden Situation entlasten und die ihnen gut tun. Bauman (1995) macht auf die Bedeutung von Ordnungsschemata im Umgang mit ambivalenten Situationen aufmerksam. So hat die Konstruktion von Polaritäten (das Ordnungsschema) die Funktion der Vereinfachung und somit der Komplexitätsreduktion, um mit sozialen Phänomenen in Interaktionen umgehen zu können: „Keine binäre Klassifikation, die in der Konstruktion von Ordnung verwendet wird, kann sich vollkommen mit der wesentlich nicht-diskreten, kontinuierlichen Erfahrung der Realität decken“ (Bauman 1995: 83). Diese Annahmen treffen ebenfalls auf die von mir untersuchten Freiwilligen zu. Für die Positionierungen und Suchbewegungen benötigen die jungen Menschen Ordnungsschemata und Sicherheiten, die sie meines Erachtens in den Konstruktionen von Bipolaritäten herstellen. Diese Konstruktionen erzeugen Sicherheit und geben ihnen Orientierung. Sowohl der Zusammenschluss mit Menschen, von denen man sich (angeblich) verstanden fühlt, als auch die Konstruktion von bipolaren Ordnungen können somit im Sinne von Bewältigungsstrategien als wichtige Ressource betrachtet werden. Sie bergen aber stets auch die Gefahr, über den Rückgriff auf stereotypes Wissen diese Stereotypen zu reproduzieren.

Steht aber nicht die Überlegung zur Bewältigung von herausfordernden Situationen im Fokus, sondern das Ziel der interkulturellen Öffnung, welches häufig mit dem Engagement im Internationalen Freiwilligendienst verbunden wird, so stellt genau diese herausfordernde Ambivalenzsituation und das aufgezeigte „Ringen um angemessenes Verhalten" eine wichtige Ressource dar. Wichtig erscheint jedoch, dass es nicht zu einer sofortigen und einseitigen Auflösung der Ambivalenz kommt, sondern dass der Irritation, welche in dem „Nichtwissen wie“ verborgen ist, Raum geschaffen wird. Die Ambivalenz - als unauflösbares Spannungsverhältnis - wird also als solche anerkannt. Dieses Ringen findet im „Inbetween“ statt, was als Zwischenraum verstanden werden kann. In diesem Zwischenraum ist das „Ringen um angemessenes Verhalten“ zu verorten.

Um die Frage nach der Chance des „Inbetween“ zu beantworten und einen Perspektivenwechsel vorzuschlagen, kann sich der Perspektive der Transnationalitätsforschung bedient werden, wie sie beispielsweise von Glick Schiller (2007) vorgeschlagen wird. Der Transnationalitätsdiskurs bietet eine Erweiterung der Perspektive „entweder-oder“ auf das „sowohl-als-auch“. Diese Erweiterungen und Verflechtungen, die 
sich über die Gleichzeitigkeit ergeben, bedeuten jedoch nicht, dass soziale Situationen ohne Polarisierungen auskommen und sich im „Trans“ auflösen.

Das „Inbetween“ kann sowohl als Ambivalenz als auch als „Trans“ gesehen werden. Indem sich die Freiwilligen in einer „Inbetween“-Situation befinden, erleben sie sowohl Ambivalenzen als auch Prozesse der „Trans“-Erfahrungen. So stellt das „Inbetween“ - je nach Perspektive - eine Herausforderung oder eine Chance bzw. eine Herausforderung und eine Chance dar. Im „Inbetween“ ergeben sich Möglichkeiten des Oszillierens zwischen den Polen. Es entsteht ein Raum der Reflexion für die Freiwilligen. So ist keine (einseitige) Entscheidung nötig. Dieser Reflexionsraum kann und muss meines Erachtens insbesondere in der pädagogischen Begleitung der Freiwilligen zum Thema gemacht und dadurch genutzt werden.

\section{Literaturverzeichnis}

Bauman, Zygmunt (1995): Moderne und Ambivalenz. Das Ende der Eindeutigkeit, Frankfurt am Main.

Fischer, Jörn (2011): Freiwilligendienste und ihre Wirkung - vom Nutzen des Engagements, in: Aus Politik und Zeitgeschichte, Heft 48/2011, S. 54-62.

Glick Schiller, Nina (2007): Transnationality, in: A Companion to the Anthropology of Politics, hrsg. von David Nugent und Joan Vincent, Oxford, S. 448-467.

Honer, Anne (1994): Einige Probleme lebensweltlicher Ethnographie. Zur Methodologie und Methodik einer interpretativen Sozialforschung, in: Interpretative Sozialforschung. Auf dem Weg zu einer hermeneutischen Wissenssoziologie, hrsg. von Norbert Schröer: Opladen, S. 85-106.

Kontzi, Kristina (2015): Postkoloniale Perspektiven auf „weltwärts“ - ein Freiwilligendienst in weltbürgerlicher Absicht, Baden-Baden.

Krettenauer, Tobias und Niki Gudulas (2003): Motive für einen Freiwilligendienst und die Identitätsentwicklung im späten Jugendalter: Eine empirische Untersuchung zur Lebenslaufcharakteristik „neuen sozialen Engagments“, in: Zeitschrift für Entwicklungspsychologie und Pädagogische Psychologie, 35. Jg., Heft 4, S. 221-228.

Lüders, Christian (2003): Beobachtungen im Feld und Ethnographie, in: Qualitative Forschung. Ein Handbuch, hrsg. von Uwe Flick, Ernst von Kardoff und Ines Steinke, Reinbek bei Hamburg, S. 384-401.

Lüscher, Kurt (2000): Die Ambivalenz von Generationenbeziehungen - eine allgemeine heuristische Hypothese, iN: Erfahrung mit Generationen-Differenz, hrsg. von Luise Winterhager-Schmid, Weinheim.

Mangold, Katharina (2013): Inbetweenness: Jugend und transnationale Erfahrungen, WeinheimBasel.

Schwinge, Brigitte (2011): Verkehrte Welten: Über die Umkehrung der Verhältnisse von Geben und Nehmen: Der weltwärts-Freiwilligendienst als Selbstbehandlung im Kulturkontakt zwischen Deutschland und Südafrika, Bonn.

Strauss, Anselm und Juliet Corbin (1996): Grounded Theory: Grundlagen Qualitativer Sozialforschung, Weinheim-Basel. 